\title{
Transferts culinaires gréco-allemands dans la Grèce du roi Othon :
}

témoignages féminins

Greek-German Culinary Experiences in Greece under King Otto: Women

Testimonies

Marie-Élisabeth Mitsou

\section{OpenEdition}

Journals

Édition électronique

URL : https://journals.openedition.org/ceb/7097

DOI : $10.4000 /$ ceb. 7097

ISSN : 2261-4184

Éditeur

INALCO

Édition imprimée

ISBN : 978-2-85831-230-6

ISSN : 0290-7402

\section{Référence électronique}

Marie-Élisabeth Mitsou, «Transferts culinaires gréco-allemands dans la Grèce du roi Othon : », Cahiers balkaniques [En ligne], Hors-série | 2016, mis en ligne le 21 mars 2017, consulté le 06 juillet 2021. URL : http://journals.openedition.org/ceb/7097 ; DOI : https://doi.org/10.4000/ceb.7097

Ce document a été généré automatiquement le 6 juillet 2021

\section{(c) (†) 8}

Cahiers balkaniques est mis à disposition selon les termes de la Licence Creative Commons Attribution - Pas d'Utilisation Commerciale 4.0 International. 


\title{
Transferts culinaires gréco- allemands dans la Grèce du roi Othon :
}

\author{
témoignages féminins
}

Greek-German Culinary Experiences in Greece under King Otto: Women

Testimonies

Marie-Élisabeth Mitsou

La nourriture d'un laboureur anglais suffirait en Grèce à une famille de six personnes. Les riches se contentent fort bien d'un plat de légumes pour leur repas ; les pauvres, d'une poignée d'olives ou d'un morceau de poisson salé. Le peuple entier mange de la viande à Pâques pour toute l'année.

(ABOUT, 1863, 41)

1 Cette observation d'Edmond About dans la Grèce contemporaine s'avère un lieu commun dans les écrits de voyage en Orient au XIX ${ }^{e}$ siècle (DUCHÊNE, 2003, 641, 663, 669 ; BERCHET, 1985, 187, 196, 217-218). Les Grecs sont sobres ; ils mangent peu, ils boivent rarement. «Un morceau de poisson salé, une poignée de piments ou d'olives amères, une tranche de khalva [...] composeraient pour la somme de trois sous, un festin de Balthazar », affirme About $(1863,351)$, en précisant que :

Les cafés d'Athènes sont pleins de monde; et à toute heure; mais les consommateurs ne prennent point de liqueurs fortes. Ils demandent une tasse de café d'un sou, un verre d'eau, du feu pour allumer leur cigarettes, un journal et un jeu de dominos : voilà de quoi les occuper toute une journée.

(ABOUT, 1863, 41)

2 Où et comment les voyageurs prenaient-ils connaissance de l'alimentation des Grecs? On les observait manger en plein air dans les champs, parfois dans des gargotes ou des khans, et le plus souvent lors de leurs fêtes, ainsi le Lundi pur, lorsque les Athéniens se réunissaient autour des colonnes du temple de Jupiter olympien ou, le $1^{\mathrm{er}}$ avril, sur l'esplanade entre le temple de Thésée et la colline de la Pnyx (BERCHET, 1985, 170-171) ; mais on passait rarement le seuil des foyers. Le pain, les olives, les oignons, les légumes et les fruits constituaient-ils l'ensemble du répertoire culinaire en Grèce après la 
formation de l'État-nation? Qu'était devenue la vieille cuisine gréco-ottomane ? Où étaient passées toutes ces gourmandises qu'a mises en rimes en 1882, dans un moment de nostalgie gourmande, le lexicographe Stéphanos Koumanoudis? Le pilav, le kapama, les dolma, les köfte, le yiahni, le musakka, l'imam baildi, le güveç? - pour ne pas mentionner les mets savoureux, plus ou moins oubliés, de son enfance (MITSOU, 1995-1996, 254-255 ; MATTHAIOU, 2002, 219-220) :

L'ayran, le hoşaf, le sapara, le boza,/Le boulghour, le kuskus, le pestil, le patça,/Le yufka, le reçel, le katmer, le tarhana,/Le pihti et le petmez, pastirma et kavurma,/L'aşure, le saint bouillon et l'omelette ${ }^{1}$.

Il est vrai que les sources grecques sur la culture alimentaire des premières décennies du royaume sont plutôt rares, même dans la littérature. Jusqu'aux années 1860, la bibliographie ne révèle que deux livres de cuisine, traduits respectivement de l'italien et du français (NOTAKER, 2002, 147 et 255) ${ }^{2}$. Point de traces de la cuisine traditionnelle ni des échanges culinaires entre les diverses populations de l'Empire ottoman dont parlent les voyageurs des siècles précédents (MAтthaiou, 1997 et 2002, 217-218). L'influence française reste d'ailleurs dominante jusqu'à la fin du siècle, vu que, comme le constate l'historienne Anna Matthaiou, l'alimentation paysanne n'avait aucun prestige aux yeux de l'élite $(2002,147)$. Les recettes françaises et italiennes dévoilent ainsi un projet de modernisation culinaire, au moins en ce qui concerne les classes privilégiées. En revanche, il semble que la culture alimentaire de l'Europe centrale n'ait pas eu d'effet sur les Grecs, malgré les trente années de Bavarocratie et les transferts culturels qui sont visibles dans plusieurs secteurs de la vie sociale et intellectuelle du royaume; ceci non seulement par l'intermédiaire du roi bavarois et de sa reine prussienne, de la cour, des hauts fonctionnaires, des architectes et archéologues, philologues et artistes, pharmaciens et médecins, enseignants et prêtres d'origine allemande, des 3500 soldats bavarois et des divers commerçants, artisans, brasseurs, cordonniers, maçons et aventuriers, qui ont cru pouvoir faire fortune dans le nouvel État du Sud-Est européen, mais aussi à travers tous ces savants grecs, qui ont occupé des postes importants dans les ministères, à l'université, dans les services publics, et qui pour la plupart avaient fait leurs études en Allemagne -comme Stéphanos Koumanoudis.

4 Trouve-t-on du bouillon et de la bonne viande en Grèce ? C'était une des premières questions posées par un Bavarois au représentant du gouvernement grec, lors de l'arrivée du roi mineur à Nauplie en janvier 1833 (DRAGoumIS, 1890, 180 ; 1973, I, 216). Pendant la Régence (1833-1835), environ 8000 Allemands se sont installés dans le royaume, dont une grande partie a dû quitter le pays après la révolution du 3 septembre 1843 (KRAUS, 1995, 183). La première colonie bavaroise fut établie en 1837 à Iraklion en Attique et dotée, depuis 1840, d'une brasserie. D'autres Allemands sont arrivés plus tard, même après l'abdication du roi Othon. Comment tous ces résidents étrangers ont-ils pu se passer de leurs habitudes culinaires et s'adapter à la sobriété grecque et aux goûts orientaux? Par des témoignages de soldats bavarois nous apprenons que leur repas quotidien contenait du pain (d'orge), de la viande (de mouton et parfois de porc), des légumes, des fruits et du vin (HILDEBRANDT, 1995, 106). Mais qu'en est-il des épouses et des femmes étrangères établies dans le pays ? Comment ont-elles $\mathrm{pu}$ adapter leurs goûts aux plats locaux? Que pensaient-elles de la cuisine des «autres»? 
5 Pour répondre à ces questions, nous avons interrogé les écrits intimes de quatre femmes de rang social différent et d'origine allemande ou scandinave, qui couvrent les années de 1834 à 1853. La première est Bettina Schinas-Savigny (Paris 1805Athènes 1835), fille $d u$ juriste et créateur de l'école d'histoire du droit Friedrich Carl von Savigny et de Gunda Brentano, qui était la nièce du célèbre écrivain romantique Clemens Brentano et de Bettina von Arnim. Bettina Savigny suivit son époux, le phanariote et futur recteur de l'université d'Athènes Constantin Schinas, à Nauplie, première capitale de la Grèce, puis à Athènes, où elle décéda dans l'été 1835 , à l'âge de trente ans, dans une épidémie de grippe typhoïde (RUTH, 2002, 7-31). Pendant son court séjour en Grèce, Bettina écrivait régulièrement à ses parents, pour leur faire part de ses aventures quotidiennes, ses excursions, ses entretiens avec le roi, avec des hommes politiques, des poètes et des anciens combattants grecs, leur parler des coutumes du peuple et de tout ce qu'en tant qu'épouse d'un Grec, elle avait l'avantage de pouvoir mieux observer que tout voyageur et tout autre étranger de passage en Grèce (RUTH, 2002, 122). Parallèlement à ces lettres, elle tenait un journal avec d'avantage de détails sur son existence journalière, qui pourraient un jour intéresser ses parents.

\section{Julie von Nordenflycht et la reine Amalia : les grandes dames}

6 Les lettres de Julie von Nordenflycht (Minden 1787-Athènes 1842), institutrice (ou «nounou» pour son entourage) de la duchesse Amélie d'oldenburg, qu'elle accompagna à Athènes après le mariage de celle-ci avec le roi Othon, sont adressées à une dame du palais d'oldenburg, son amie von Scheele, et datent de 1837 à 1842 (NORDENFLYCHT, 1922). Au début de son séjour à la cour d'Athènes, Nordenflycht avait 51 ans, mais, malgré son âge avancé, elle n'a jamais renoncé à accompagner la reine dans ses longues promenades à cheval, lors de ses excursions aux alentours de la capitale et de ses voyages à travers le pays; elle participait à toutes les fêtes du palais, aux bals, aux dîners, aux cérémonies et aux spectacles. C'était une femme cultivée, précédemment écrivaine et traductrice de Lord Byron, qui ne put jamais s'accoutumer à la vie athénienne. Aux dîners diplomatiques elle préférait ses lectures, et de tous les Grecs qu'elle a connus, elle n'appréciait que Rosa Botsari ${ }^{3}$ et les Phanariotes. Ses connaissances du grec sont restées rudimentaires. Elle est morte de la grippe en juillet 1842 .

7 Les lettres de Julie von Nordenflycht complètent en quelque sorte celles de la reine Amélie (Oldenbourg 1818-Bamberg 1875) à son père, le duc August von Oldenburg, par lesquelles la cour d'Oldenbourg s'informait de l'actualité politique en Grèce. Les 533 lettres de la reine couvrent une période qui va de son arrivée à Athènes, en février 1837, à la mort de son père, en février 1853 (BUSSE, 2011, I-II). Elles sont longues et détaillées, pleines de tendresse pour son père bien-aimé, doux, gentil, angélique ${ }^{4}$. La reine se voulait plus Grecque que les Grecques, elle se déclarait amoureuse de la Grèce et se croyait aimée par son peuple (BUSSE, 2011, I, 705, 826, 850). Après l'établissement de la Constitution, ses lettres portent principalement sur la politique, sur les intrigues des ambassadeurs des Grandes puissances et les conflits internes. 
8 La reine avait près d'elle un pasteur protestant, Asmus Heinrich Friedrich Lüth, originaire du Holstein. Celui-ci s'installa à Athènes en automne 1839, avec sa femme danoise, Christiane Lüth (Gurre/Nordsjaelland, 1817-1898), âgée alors de 22 ans, et sa belle-sœur, Hanne. Ils ont vécu dans cette ville pendant treize ans, Christiane y a accouché de ses quatre enfants, dont deux sont morts en bas âge. Elle tenait un journal et un carnet de notes, qui ont paru en partie, en 1929, au Danemark et plus récemment dans une édition intégrale en grec (PAPANIKOLAOU-CHRISTENSEN, 1988 et 1991). Ayant appris la langue du pays, Christiane se sentait plus proche des Grecs que les autres étrangères. Ses écrits sont une source précieuse sur la société entre résidents étrangers, sur les représentations que ceux-ci avaient des Grecs, mais aussi sur les soins du ménage, la variété et les prix des produits alimentaires (PAPANIKOLAOU-CHRISTENSEN, 1991, 191-195).

9 Comme on l'imagine, ni la reine ni la dame du palais ne s'amusaient à décrire ce qu'elles mangeaient dans leurs lettres. Pour Julie von Nordenflycht le proverbe « Dismoi ce que tu manges, je te dirai ce que tu es » n'avait pas beaucoup de sens. Elle lui préférait une version plus mondaine: «Dis-moi ce que tu portes, je te dirai d'où tu viens ». Ainsi on trouve dans ses écrits d'innombrables descriptions de robes en soie et de voiles en mousseline, d'écharpes, de châles turcs, de dentelles et de chapeaux, venus de Paris, de Trieste, d'Alexandrie mais aussi vendus dans les nouvelles boutiques athéniennes (NORDENFLYCHT, 1922, 434). Les habits les plus remarquables étaient portés par les femmes des diplomates, car les Grecques, prétendait-elle, s'habillaient mal : elles mettaient trop de couleurs mal associées. Par contre leurs costumes nationaux étaient bien plus intéressants, en particulier celui de l'île d'Hydra, d'autant plus qu'ils étaient commodes: les femmes n'avaient pas besoin de renouveler leur garde-robe, puisque le même costume servait de tenue de cérémonie, de soirée et de sortie. En revanche, la grande dame de la cour devait parfois se changer sept fois au cours d'une seule journée (1922, 410 et 415).

10 Julie von Nordenflycht parle beaucoup des dîners du palais. Dans sa première lettre, datée du 16 février 1837, elle décrit en détail la grande salle ronde du palais provisoire, où le couple royal avait reçu ses 50 invités : les murs et le plafond étaient revêtus de soie rouge avec une étoile au centre et les rideaux étaient également rouges avec des franges rouges et blanches ${ }^{5}$. Sous la lumière de cinq grands lustres, la table royale resplendissait avec ses vases, ses chandeliers, ses verres et ses assiettes en argent (NORDENFLYCHT, 1922, 389-390). Pourtant le contenu de ces assiettes nous reste inaccessible. En outre, il apparaît que la reine offrait deux fois par semaine un dîner aux étrangers éminents et des petits repas uniquement à des Grecs (NORDENFLYCHT, 1922, 397 ; BUSSE, 2011, I, 123 et 484) ; on voit qu'on donnait souvent de grands bals, à 500 ou 600 invités, au palais mais aussi dans les villas des ambassadeurs ou sur des bateaux. Ils commençaient vers 21 heures et duraient jusqu'à l'aube ; on y dansait la polonaise, la valse allemande et française, la mazurka, la polka et le cotillon (NORDENFLYCHT, 1922, 391-392 ; cf. BUSSE, 2011, I, 682 et 698-700) ${ }^{6}$. Mais malheureusement les thés n'étaient pas courants - dans les lettres d'Amélie, il n'est question que de thés dansants (NORDENFLYCHT, 1922, 435 ; BUSSE, 2011, I, 210).

11 Parfois on organisait des repas en plein air : on dînait sur le Pentélique ou l'Hymette, près de monastères, entre oliviers et pins, sous des platanes ou sous une pergola couverte de feuilles de myrtes et de lauriers roses, on servait des plats préparés sur place avec des provisions apportées de la ville, on buvait du champagne et on dansait 
(NORDENFLYCHT, 1922, 407 et 441; cf. BUSSE, 2011, I, 102). On fêtait l'anniversaire du roi, celui de ses noces, de son arrivée à Nauplie, l'anniversaire de la reine, Noël, le Nouvel An, le carnaval et le 25 mars (NORDENFLYCHT, 1922, 412-415, 459, 461 et 489). D'habitude le couple royal dînait vers 19 heures. Mais il lui arrivait parfois de prendre le petit déjeuner à midi, déjeuner à 16 heures et souper à minuit (NORDENFLYCHT, 1922, 445). Aux dîners des diplomates, le roi et la reine étaient toujours servis à part ; assis à la même table, les maîtres de maison restaient à jeun pour le plaisir de regarder leurs hauts invités savourer leur repas (BUSSE, 2011, I, 366-367).

Or, ni la reine ni la grande dame de la cour ne se souciaient de ce que mangeait le peuple. Même pendant leurs tournées dans le pays, elles n'ont jamais saisi l'occasion de goûter, comme la Comtesse de Gasparin, des plakountes ou pâtes salées, que les paysannes faisaient cuire au four et qui datent, paraît-il, de l'Antiquité (BERCHET, 1985, 217 ; MATTHAIOU, 2002, 224 ; NORDENFLYCHT, 1922, 429-431 et 507-513). À la rigueur, elles déjeunaient sur l'herbe, se contentant de plats froids (de pain, d'œufs, de poulet, de fromage et de chocolat), parfois elles goûtaient l'agneau rôti et le lait qu'on leur offrait dans les monastères (BUSSE, 2011, I, 113, 167, 172, 660, 664-666 et 765). Une fois, en automne 1844, le couple royal s'est permis de dîner dans le village d'Éleusis et de danser avec les villageois. La danse a vite tourné à la valse, les paysans suivaient de loin ; la reine a offert des bonbons aux petits (BUSSE, 2011, I, 649-650 et $763 \mathrm{sv}$.).

13 En janvier 1840, on a fêté l'inauguration du palais royal, dont les travaux ne seront terminés qu'au bout de trois ans ${ }^{7}$. Ce jour-là les ouvriers ont eu droit à cinquante agneaux à la broche et 4000 bouteilles de vin (NORDENFLYCHT, 1922, 492). Mais pour le reste, on imagine, avec Edmond About, qu'ils se contentaient d'une poignée d'olives marinées dans la saumure et de pain (ABOUT, 1863, 98). La reine Amélie fut profondément impressionnée par la fête populaire du premier jour du carême (BUSSE, 2011, I, 69). Tout était très pittoresque : les costumes, les colonnes, l'ambiance - on dirait un tableau de Christian Perlberg8. Elle raconte à son père que ses sujets avaient l'habitude de prendre en plein air leur repas simple, composé d'oignons, de pain et d'olives; qu'ils étaient tous de bonne humeur, vêtus de leurs costumes locaux, dansant en rond et admirant leurs chefs militaires qui marchaient fièrement le long du site archéologique ; qu'un Grec a offert au roi un flacon en bois avec du vin (BUSSE, 2011, I, 157).

Décidément la reine n'était pas gourmande, aussi préférait-elle tromper sa faim avec des oranges plutôt que goûter des poulpes qu'elle traitait de monstres de mer (BUSSE, 2011, I, 767). Elle mangeait rarement du poisson. Du reste elle adorait les fruits doux, surtout les figues et les raisins (BUSSE, 2011, I, 90 et 102) et ne se plaignait pas, comme About, de « la malheureuse habitude [des Grecs] de cueillir les fruits avant qu'ils soient murs » $(1863,103)$. Mais elle regrettait le bon beurre de son pays. Elle envoyait à son père des dattes séchées, du vin de Santorin et des loukoums. De son côté, il la récompensait par des airelles (BUSSE, 2011, I, 378 et 733). Quittant définitivement la Grèce, en octobre 1862, après une dernière tournée dans le Péloponnèse, la reine emporta dans ses bagages son costume national, connu sous le nom d'Amélie, qu'elle portait parfois dans sa résidence d'exil à Bamberg. Ses dernières années sont empreintes de la nostalgie d'un pays auquel elle s'était profondément attachée nostalgie de sa nature, de ses traditions, mais pas nécessairement de sa cuisine. 


\section{Bettina Schinas et Christiane Lüth : deux bourgeoises}

15 Contrairement aux grandes dames, les deux bourgeoises, Bettina Schinas et Christiane Lüth, avaient un goût très vif pour la culture culinaire de leur patrie adoptive - la première par curiosité intellectuelle, la seconde pour des raisons pratiques. Dans sa deuxième lettre, depuis Patras, Bettina enregistrait déjà son premier repas en Grèce avec du poisson grillé ainsi que la bonne manière de goûter les fruits au

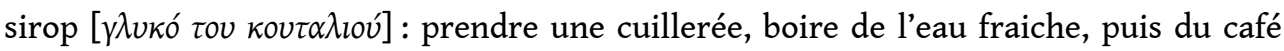
(RUTH, 2002, 46). Dans l'intention d'adopter la cuisine grecque, elle dresse une liste des plats familiers : soupe, olives, gigot d'agneau, poissons, ragoût, poulet farci, céleri, pommes de terre, tranches de viande avec du foie haché, des amandes, des raisins secs et des épices, sablés. Le pain blanc et noir, écrit-elle, préparé autrement que chez nous, est délicieux : il se laisse conserver pendant trois jours sans devenir dur, ce qui est assez bizarre. L'huile d'olive, le vin de Céphalonie, les fruits locaux sont exquis. En été les repas devenaient plus légers : il fallait surtout éviter la viande, les limonades et les fruits acides (RUTH, 2002, 55).

Pendant son séjour temporaire à Nauplie, Bettina fut impressionnée par le nombre de boutiques d'alimentation, où on pouvait se procurer toutes sortes de viande, de gibier, de poissons, de fromages et de légumes: choux, carottes, navets, épinards, endives, haricots, chou-raves, laitues, persil, herbes sauvages comestibles, herbes aromatiques, oignons, tomates, melinsanes (aubergines, qu'on ne connaissait pas encore en Allemagne). Des fruits frais en quantité : pommes, raisins, grenades, agrumes, coings, et des fruits secs pour tous les gouts - figues, dattes, raisins. Il ne manquait rien sur le marché : on y trouvait du riz, des fèves, des œufs, de la farine, du lait, du chocolat, du thé, des gâteaux. Quant aux prix, ils avaient grimpé depuis le jour où la Régence avait décidé de se déplacer à Athènes (RUTH, 2002, 69).

La jeune Berlinoise avait au début une cuisinière allemande, puis un cuisinier grec, mais elle leur aurait préféré une femme hydriote. Malgré tout, elle était ravie de ses repas: au petit déjeuner elle prenait du chocolat chaud et du pain; un deuxième petit déjeuner chaud suivait à midi ; on déjeunait vers 17 heures et on dînait à 20 heures (RUTH, 2002, 81). Ainsi, elle pouvait rassurer sa maman et affirmer que, dans ce pauvre pays du Sud de l'Europe, elle se sentait mieux que jamais. Elle fréquentait surtout des Allemands et des proches de son mari, les Soutsos, les Rizos, les Rangabé ; on prenait du thé, on dînait ensemble, on se promenait dans la campagne. Bettina notait soigneusement des recettes de mets et de gâteaux et rêvait déjà de sa nouvelle demeure athénienne que lui avait promise le régent Carl Wilhem von Heideck: située sur la route de Pirée, loin des ruines de la ville dévastée par la guerre, elle aurait un jardin et une vue splendide sur Égine, le port, l'oliveraie, les montagnes et même sur l'Acropole (RUTH, 2002, 126 et 134).

Ce que Bettina découvrit en avril 1835, après une journée de voyage en bateau et une heure et demie de mauvaise route depuis le Pirée, ne correspondait pas exactement à ses désirs : la maison qu'on avait louée pour eux se trouvait au creux du bassin, à une demi-heure de marche de la ville, où on allait chercher l'eau et des provisions, elle était exposée au vent, à la poussière, à l'humidité et à la chaleur, sans ombre, sans vue, avec une cuisine construite à moitié et séparée de l'habitation, et une vaste pièce, dans laquelle on dormait, on vivait et on déjeunait, avec des souris, des moustiques et des puces (RUTH, 2002, 183 et 190-191 ; MITSOU, 2005, 32-33). Douée d'une admirable patience, 
Bettina ne se lassait pourtant pas de recevoir dans cette ambiance modeste des comtes et des ambassadeurs, Ioannis Coletti et d'autres amis de famille, dans l'espoir, il est vrai, de pouvoir déménager au plus vite (RUTH, 2002, 203 et 239). Elle est morte dans cette même maison en août $1835^{9}$.

Quatre ans plus tard, lorsque la famille Lüth débarqua au Pirée, les conditions de vie dans la capitale s'étaient beaucoup améliorées. Le chemin du port à la ville était bien plus court et on pouvait facilement trouver une demeure convenable, surtout si on avait l'aide de Julie von Nordenflycht et un salaire annuel de 4300 drachmes (PAPANIKOLAOU-CHRISTENSEN, 1988, 27-29; 1991, 12) ${ }^{10}$. Ainsi, les Lüth se sont logés avec leurs domestiques dans une maison en face du palais royal. Cependant le problème de Christiane Lüth était d'un tout autre ordre; en effet, pour des raisons obscures, son mari avait l'habitude de changer de demeure tous les ans. Ce déménagement perpétuel a tourné au cauchemar, dès que la famille s'est agrandie. Il fallait chaque fois emballer tous les ustensiles du ménage, transporter les meubles, les enfants, les poules, le chat, les chiens, la tortue, et s'attendre à de nouveaux inconvénients : les chambres étaient trop étroites, les voisins bruyants et indiscrets, les cigales de la cour assourdissantes. En outre, dès la première chaleur, Asmus Lüth était saisi du désir ardent de fuir la ville. On chargeait alors la calèche des enfants et des biens, on louait des ânes et on partait en vacances dans le monastère de Kaisariani, où une colonie d'Athéniens, grecs et étrangers, occupaient des chambres, partageaient les repas et la cuisine, se disputaient et médisaient sur les affaires de ceux qui avaient déjà quitté les lieux (PAPANIKOLAOU-CHRISTENSEN, 1988, 45-48, 73 ; 1991, 26-34, 40-56, 62). Parfois le pasteur de la reine changeait de plan au bout de quelques jours ; alors on remettait en caisse ses effets personnels et les ustensiles de cuisine et on rentrait chez soi pour repartir bientôt en croisière avec un caïque ou le yacht royal (PAPANIKOLAOU-CHRISTENSEN, 1988, $61,169 \mathrm{sv}$.$) .$

Christiane s'est très vite accoutumée à cette vie vagabonde. Elle parlait couramment le grec et elle a su nouer des relations avec les lettrés et les artistes les plus éminents de la capitale $^{11}$. Tandis qu'elle s'occupait du ménage, ses deux enfants s'amusaient dans la rue avec leurs petits voisins et se taquinaient en grec vulgaire. Les récits de Christiane Lüth sur les plats et les produits alimentaires consommés offrent une vue d'ensemble des habitudes et des échanges culinaires des familles aisées en Grèce du XIX ${ }^{e}$ siècle (PAPANIKOLAOU-CHRISTENSEN, 1991). On se régalait de pâtes italiennes, de poissons et de fruits de mer, de lièvre farci, d'agneau et de sanglier, de volaille et de gibier, de pilav et de riz, de marides et de kolokythakia, de viande de veau vedello et maïatiko, de keftedes et de khalva, on buvait du lait de brebis bouilli, du café cuit à la turque, du salep, de la bière, du vin (de Santorin, de Syros, de Chypre et de la malvazia/ Malvoisie), du champagne, du raki et des liqueurs, on préparait du yaourt, des fruits au sirop (prunes, abricots, coing), des beignets, des diples et des koulourakia, on allait chercher au marché des huîtres, de la semoule de blé dur, des biscuits américains, du miel et des koulouria (PAPANIKOLAOU-CHRISTENSEN, 1991 ; les mots en italique sont en grec dans le texte).

21 La jeune Danoise nous renseigne sur les prix de tous les produits en vente dans les boutiques athéniennes et sur le coût des services, sans oublier de petits détails amusants: la femme de l'architecte du roi, Riedel, était une grosse mangeuse; en revanche la duchesse de Plaisance se nourrissait d'eau et de sardines ; le domestique de la voisine leur offrait généreusement des œufs volés au poulailler de celle-ci ; le moine 
de Kaisariani a goûté leur plat de viande tout en regardant furtivement derrière lui, car c'était le moment du carême ; le mari de Christiane, parti à la chasse, est rentré le soir avec des perdrix qu'il avait achetées chez le boucher du quartier ; la reine a fait cadeau aux enfants des Lüth d'un petit agneau qu'on lui avait offert; les Bavarois qui fréquentaient la Cour étaient pour la plupart des escrocs à éviter ; les Grecs dévoraient leurs œufs de Pâques dans l'église, dès que minuit sonnait, en jetant les coques par terre ; aux bals du palais, on ne servait aux invités que des gâteaux et de la limonade, ce qui exaspérait les Allemands; les recettes de cuisine danoise, comme celle de Christiane Rosen, appliquées aux produits grecs, ne valaient rien, etc. (PAPANIKOLAOUCHRISTENSEN, 1988, 72, 106, 115, 145, $147 ; 1991,33,56,71,95,134)$.

\section{Conclusion}

Pour conclure, les quatre témoignages féminins $d u \mathrm{XIX}^{\mathrm{e}}$ siècle permettent certaines réflexions. Il semble tout d'abord que la culture culinaire n'est pas un privilège de la haute société ; elle s'avère plutôt une question de goût, de curiosité intellectuelle et de générosité. Par ailleurs, la bonne cuisine concerne aussi bien les amateurs et les gourmets que des hommes et des femmes ordinaires: des épouses et des mères affectueuses, des voyageurs accommodants, des esprits ouverts. S'attendre à découvrir des secrets culinaires dans les 2000 pages des lettres de la reine Amélie et dans celles de sa dame de compagnie serait une illusion. Les intérêts de ces femmes portaient sur d'autres aspects de la vie mondaine et sociale, plus directement liés au pouvoir. Aussi, toutes deux sont restées obstinément attachées à leurs traditions nationales et n'ont cherché dans leur pays adoptif que la confirmation de leurs lectures philhelléniques et des clichés orientalistes. En revanche, Bettina Schinas-Savigny et Christiane Lüth étaient vivement intéressées par la cuisine locale ; elles étaient disposées à explorer les habitudes alimentaires d'un peuple étranger, à adopter ses coutumes et à échanger leurs propres expériences. Heureusement, les écrits intimes des deux bourgeoises nous rassurent sur le fait que la culture culinaire, en particulier celle de la Méditerranée, était depuis toujours et avant tout un plaisir à partager.

\section{BIBLIOGRAPHIE}

ABOUT Edmond, 1863, la Grèce contemporaine, Paris : Hachette.

BERCHET Jean-Claude (dir.), 1985, le Voyage en Orient : anthologie des voyageurs français dans le Levant au XIX siècle, Paris : Robert Laffont.

BRUEGEL Martin \& LAURIOUX Bruno (dir.), 2002, Histoire et identités alimentaires en Europe, Paris : Hachette.

DUCHÊNE Hervé, 2003, le Voyage en Grèce : anthologie du Moyen Âge à l'époque contemporaine, Paris : Robert Laffont. 


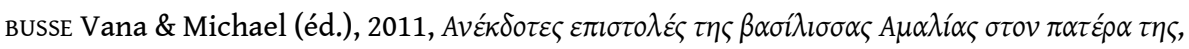
1836-1853 [Lettres inédites de la reine de Grèce Amélie à son père, 1836-1853], I-II, Athènes : Estia. DRAGOUMIS Nikolaos, 1890, Souvenirs historiques : Capo d'Istria - La Régence - Le règne d'Othon, trad. par Jules Blancard, Paris : Firmin-Didot et Cie.

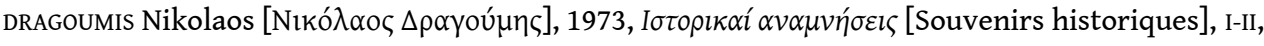
Athènes : Ermis.

HEYDENREUTER Reinhard, MURKEN Jan, wÜNSCHE Raimund (dir.), 1995, Die erträumte Nation: Griechenlands Wiedergeburt im 19. Jahrhundert [la Nation rêvée : la renaissance de la Grèce au $\mathrm{XIX}{ }^{\mathrm{e}}$ siècle], Munich : Biering \& Brinkmann.

HILDEBRANDT Maria, 1995, «"Jetzt fahren wir ins Griechenland”: Bayerische Soldaten in Griechenland in Tagebüchern und Volksliedern» [ "Maintenant nous partons pour la Grèce" : des soldats bavarois en Grèce à travers leurs journaux et chants populaires »], in HEYDENREUTER et al., Die erträumte Nation: Griechenlands Wiedergeburt im 19. Jahrhundert [la Nation rêvée : la renaissance de la Grèce au XIX ${ }^{\mathrm{e}}$ siècle], op. cit., p. 103-111.

KRAUSS Marita, 1995, »Das Ende des Traumes - bayerisches Flüchtlingselend in Griechenland« [ « La fin du rêve - La misère des refugiés bavarois en Grèce »], in HEYDENREUTER et al., Die erträumte Nation: Griechenlands Wiedergeburt im 19. Jahrhundert [la Nation rêvée : la renaissance de la Grèce au XIX ${ }^{\mathrm{e}}$ siècle], op. cit., p. 183-190.

MATTHAIOU Anna, 1997, Aspects de l'alimentation en Grèce sous la domination ottomane : des réglementations au discours normatif, Francfort/M : Peter Lang.

MATTHAiou Anna, 2002, "Prier comme un Turc et manger comme un chrétien" : la circulation des cultures alimentaires sous la domination ottomane ", in BRUEGEL \& LAURIOUX, Histoire et identités alimentaires en Europe, op. cit., p. 217-228 et 267-269.

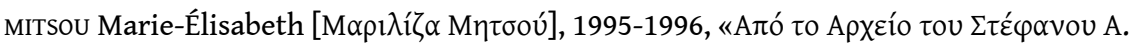

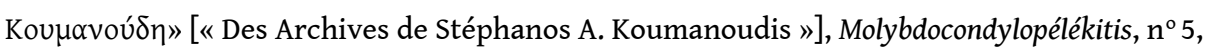
p. 249-255.

MITSOU Marie-Élisabeth, 2005, »Bruchstücke des Alltagslebens im Athen König Ottos: Die Tagebücher und Briefe von Christiane Lüth und Bettina Schinas-Savigny« [« Scènes de la vie quotidienne à Athènes du roi Othon : les journaux et les lettres de Christiane Lüth et Bettina Schinas-Savigny »], in H.-D. BLUME et C. LIENAU (dir.), Der fremde und der eigene Blick: Reisen und Reisende in Griechenland [Regard étranger et regard de soi : voyages et voyageurs en Grèce], Choregia $\mathrm{n}^{\circ} 4$, p. 25-41.

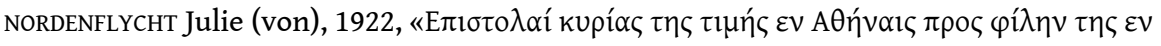

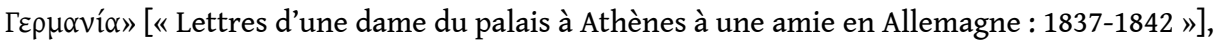
trad. Constantinos TSAOUSOPOULOS, Deltion tis istorikis kai ethnologikis etairias tis Ellados, t. 8, 1922, p. 384-554 [éd. originale : Briefe einer Hofdame in Athen an eine Freundin in Deutschland. 1837-1842, Leipzig, Hinrichs, 1845].

NOTAKER Henry, 2002, « En contrepoint : l'identité nationale à travers les livres de cuisine du XIX ${ }^{\mathrm{e}}$ siècle ", in BRUEGEL \& LAURIOUX, Histoire et identités alimentaires en Europe, op. cit., p. 137-150 et 254-256.

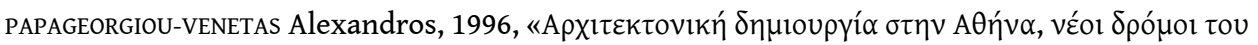

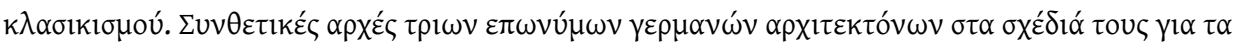

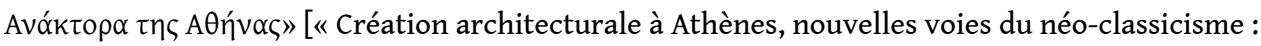


les principes de trois architectes renommés allemands dans leurs plans du palais royal

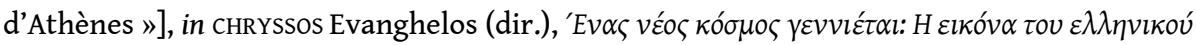

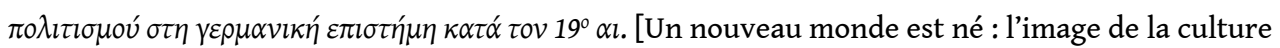
grecque dans la science allemande du XIX ${ }^{\mathrm{e}}$ siècle], Athènes : Akritas, p. 277-314.

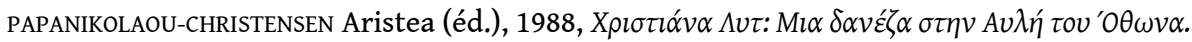

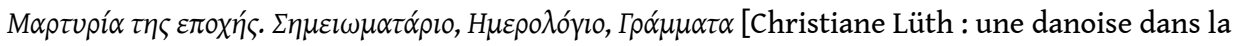
cour du roi Othon. Témoignage d'une époque. Carnet de notes, Journal, Lettres], Athènes : Ermis.

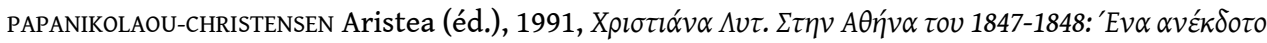
$\eta \mu \varepsilon \rho o \lambda o ́ \gamma ı$ [Christiane Lüth, Athènes des années 1847-1848 : journal inédit], Athènes : Ermis.

RUTH Steffen (éd.), 2002, Leben in Griechenland 1834 bis 1835 Bettina Schinas, geb. von Savigny: Briefe und Berichte an ihre Eltern in Berlin [Vie en Grèce 1834-1835 : Bettina Schinas, née von Savigny. Lettres et rapports à ses parents à Berlin], Münster : Éd. Cay Lienau.

TSIOMIS Yannis, 1985, Athènes affaire européenne, Athènes : Ministère de la Culture (en grec et en français).

\section{NOTES}

1. Ayran : lait caillé, qui se boit glacé avec du sel et de la menthe ; hoşaf: compote de fruits secs bouillis avec du sucre ; sapara : sorte de boisson ; boza : boisson fermentée à base de céréales; boulghour: blé bouilli, séché et concassé; pestil : pâte de fruits en feuilletée ; patça : panse et pieds d'agneau bouillis ; yufka : feuilleté ; reçel : marmelade de fruits confits moelleux dans du sirop; houssmeri: sorte de pita; tarhana: orge concassée au lait caillé ; pihti : gelée de porc ; petmez : produit tiré du moût de raisin bouilli ; pastirma : viande de bœuf fortement pressée et séchée à l'air ; kavurma : viande d'agneau rissolée avec du beurre et des oignons ; aşure : jus de blé, de farine et de sucre avec des raisins secs et des amandes ou des pistaches; saint bouillon: bouillon

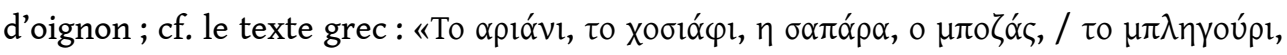

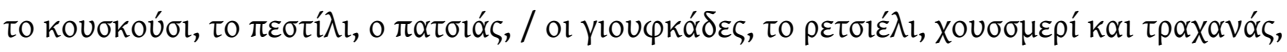

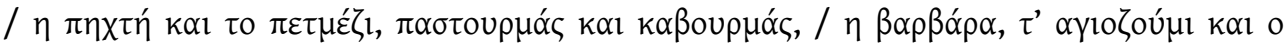

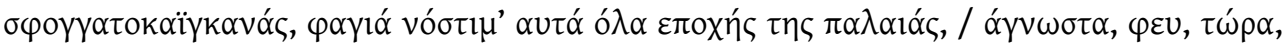

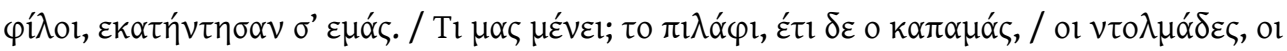

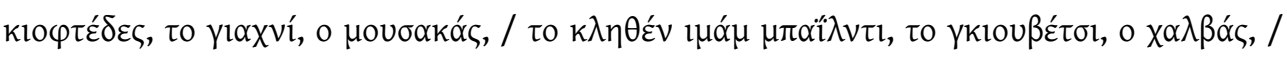

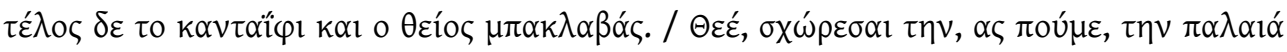

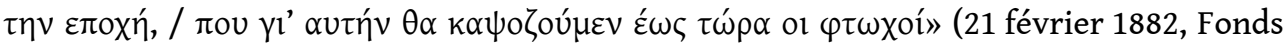
Koumanoudis, BNG, F 1140 22).

2. Il s'agit respectivement des ouvrages: la Cuisine, trad. de l'italien [par

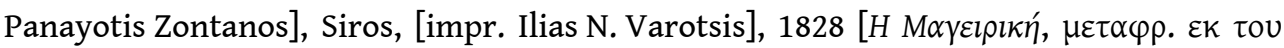

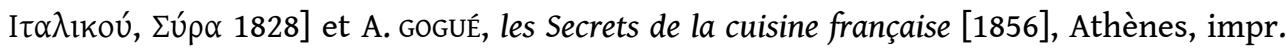

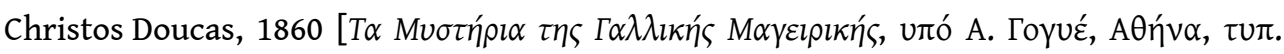

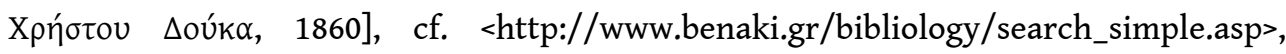
consulté le 17 décembre 2015.

3. Catherine-Rosa Botsari-Karatza (1818-1875), fille de Markos, fut nommée en 1838 dame d'honneur de la reine Amélie. Son portrait, peint par Joseph Karl Stieler, fait partie de la Galerie des Beautés du Château de Nymphenbourg à Munich. 
4. C'est par ces mots que la reine Amélie s'adressait à son père : $\alpha \gamma \alpha \pi \eta \mu \varepsilon ́ v \varepsilon, k \alpha \lambda \varepsilon^{\prime}, \gamma \lambda u \kappa \varepsilon$,

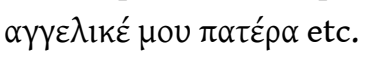

5. Le palais provisoire (1836-1843) a été l'une des premières maisons construites à Athènes d'après les plans des architectes allemands G. Lüders et J. Hoffman, la propriété de Dekozi-Vourou à la place Klafthmonos, qui abrite aujourd'hui le Musée de la ville d'Athènes.

6. Aux bals du palais étaient invités tous les diplomates, les officiers de la marine étrangère, les sénateurs, les ministres, les députés, les hauts fonctionnaires et les officiers grecs ainsi que la noblesse. Edmond About ne se lasse pas de commentaires ironiques sur ces soirées : «Ces bals de la cour se composent presque exclusivement de valses et de contredanses. On valse à deux temps. La valse à deux temps, je ne sais pourquoi, s'appelle en Grèce la valse allemande. La valse à trois temps est indûment qualifiée de valse française. [...] Vers le milieu du bal on donne une polka, une seule. La polka est la danse favorite du roi ; mais la reine ne peut pas la souffrir. [...] On n'a pas encore entendu parler de la varsoviana. [...] En revanche, on danse toujours une mazurka à grands ramages, avec figures, et l'on termine régulièrement par un interminable cotillon » $(1863,321)$; « Les bals se terminent à trois heures du matin ; ils durent donc six heures, dont deux au moins se passent en conversation. L'éclairage de la salle de danse est très brillant; les rafraichissements le sont beaucoup moins. Les gâteaux qu'on fait passer sont presque tous des pains d'épices déguisés. On se bat à la fin pour avoir du bouillon » (ibid., 322 ; cf. 310, 319-320).

7. Le palais royal - où siège depuis 1935 du Parlement grec - a été construit d'après les plans de l'architecte Friedrich von Gärtner ; sur l'histoire du projet du palais royal, cf. TSIOMIS, 1985, 91-102 et PAPAGEORGIOU-VENETAS, 1996.

8. Le tableau de Christian Perlberg (1806-1884) Lundi pur à Athènes (1838) est exposé au Musée national historique d'Athènes, <http://www.nhmuseum.gr>. Cf. le commentaire d'Edmond About sur cette fête populaire : « Le lundi, tout le peuple d'Athènes se réunit autour des colonnes du temple de Jupiter, pour commencer en commun les mortifications de quarante jours. Il s'y fait une grande consommation d'ail, d'oignon et de toutes sortes de légumes crus. On chante beaucoup, et du nez; on boit un peu, on ne danse pas mal. Après cette cérémonie chacun rentre chez soi. Je ne sais rien de plus propre à aigrir le sang que le carême des Grecs. Non seulement ils se privent de viande, mais ils s'interdisent le beurre, les œufs, le sucre et souvent le poisson. Ils ne mangent que du pain, du caviar et des herbes assaisonnés d'un peu d'huile» $(1863,246$; cf. ibid. 248 ; PAPANIKOLAOU-CHRISTENSEN, 1988, 111 et 1991, 164).

9. Sur le monument funéraire de Bettina dans le cimetière des protestants à Athènes (premier cimetière), inspiré par un dessin de Stamatios Kléanthis, voir l'article d'olga FOUNDOULAKI, <http://www.archaiologia.gr/wp-content/uploads/2011/07/117-13.pdf>, consulté le 17 décembre 2015.

10. Le loyer mensuel de leur habitation principale coûtait entre 70 et 100 drachmes (PAPANIKOLAOU-CHRISTENSEN, 1991, 201). Dans les années 1840, la population d'Athènes ne dépassait pas les 30000 habitants (PAPANIKOLAOU-CHRISTENSEN, 1988, 156).

11. Les Lüth fréquentaient entre autres les professeurs Constantinos Fréaritis et Philippos Ioannou, les architectes Édouard Schaubert, Hans Christian et Théophile Hansen, les archéologues Ernst Curtius, Ludwig Ross et Erik Ulrich, le colonel 
Christoph Heinrich Fabricius, les écrivains Emanuel Geibel et Hans Christian Andersen, le théologien américain John Hill, etc.

\section{RÉSUMÉS}

Cette contribution examine les expériences culinaires de quatre femmes d'origine allemande et danoise dans la Grèce du roi Othon. Dans leurs écrits intimes (1834-1853) elles dépeignent leurs soucis quotidiens et leurs efforts pour s'adapter aux produits alimentaires et aux goûts orientaux d'un pays pauvre de l'Europe du Sud.

This paper examines the culinary experiences of four foreign women living in Greece under the rule of King Otto. In their letters and diaries (1834-1853) they describe their daily tribulations and their efforts to adapt to the available produce and tastes of a poor, semi-oriental country of South Europe.

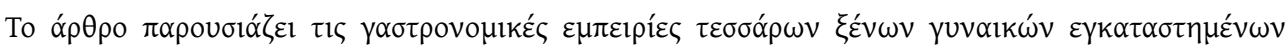

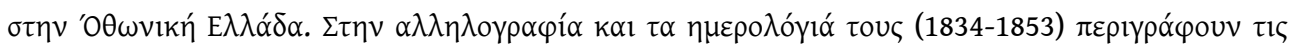

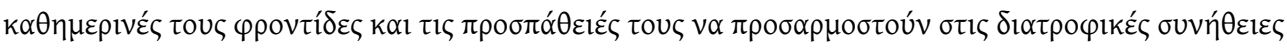

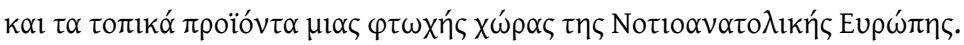

\section{INDEX}

\section{Index géographique : Grèce}

Keywords : Cultural transfer, Food culture, Tradionnal Cooking, Local Produce, Diaries, Travellers, Bavarocratia, Diner, Ceremony, Popular holiday

Thèmes : Histoire sociale, Histoire culturelle

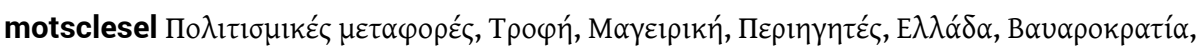

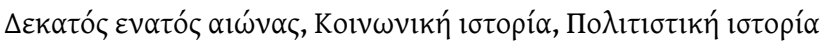

Mots-clés : transferts culturels, culture alimentaire, culture alimentaire, transferts culturels, cuisine traditionnelle, produits locaux, produits locaux, écrits intimes, écrits intimes, Bavarocratie, Bavarocratie, voyageurs, voyageurs, dîners, dîners, cérémonies, cérémonies, fêtes populaires, fêtes populaires

motsclesmk ХРАНА, ГАСТРОНОМИЈА, КУЛТУРНИ ТРАНСФЕРИ, ПАТЕПИСИ, ГРЦИЈА, БАВАРСКАТА ОКУПАЦИЈА ДЕВЕТНАЕСЕТТИОТ ВЕК, СОЦИЈАЛНА ИСТОРИЈА, КУЛТУРНА ИСТОРИЈА motsclestr Yiyecek, Gastronomi, Kültürel transferler, Gezi, Yunanistan, Bavyeran'ın işgalı, Ondokuzuncu yüzyıl, Sosyal tarih, Kültürel tarih

Index chronologique : dix-neuvième siècle

\section{AUTEUR}

MARIE-ÉLISABETH MITSOU

CRH, EHESS, Paris 